\title{
The biomechanics of iatrogenic spinal destabilization and implant failure
}

\author{
Richard P. Schlenk, M.D., Todd Stewart, M.D., And Edward C. Benzel, M.D. \\ Cleveland Clinic Spine Institute, Cleveland Clinic Foundation, Cleveland, Ohio
}

\begin{abstract}
Revision spinal surgery is usually indicated in cases of persistent or recurrent symptoms related to neural compression, spinal deformity, or construct failure. An understanding of fundamental biomechanical principles of both spinal decompression and reconstructive strategies is essential to avoid unnecessary subsequent spinal operations.
\end{abstract}

KEY WORDS • biomechanics • revision surgery • spinal destabilization

The chief objectives of spinal surgery are the decompression of neural elements, correction of existing spinal deformity, and stabilization of dysfunctional spinal motion segments. Spine surgery that has failed, however, often is a result of the potential destructive and destabilizing effects of surgery. While attempting to gain adequate exposure for proper neural decompression, aggressive and often unnecessary excision of spinal ligaments and bone may cause significant loss of spinal integrity requiring secondary surgery to reestablish stability. In addition, surgical exposure causes significant muscle injury and muscle denervation resulting in dysfunction of those muscle fibers, which may significantly contribute to unsuccessful outcomes. These factors should be considered during the surgery-related decision-making process. Pathological or iatrogenic reduction in stability, if biomechanically significant, must be compensated for by one or a combination of three therapeutic maneuvers: 1) postural, nonoperative management (including spinal splinting) that provides time for osseous and ligamentous healing to offset acute disruption of spinal integrity; 2) ventral spinal bone strut or instrumentation placement; and 3) dorsal instrumentation placement.

An understanding of the biomechanical properties of the spinal ligamentous and osseous structures is important for the surgeon to avoid causing destabilization. In addition, it is important to have a clear understanding of the biomechanics of constructs and why they fail. Construct failure typically occurs when the implant, implant-bone interface, or component-component juncture fails. ${ }^{16,22}$

\footnotetext{
Abbreviations used in this paper: $\mathrm{ALL}=$ anterior longitudinal ligament; IAR = instantaneous axis of rotation; PLL = posterior longitudinal ligament; VB = vertebral body.
}

\section{VENTRAL SURGICAL SPINAL DESTABILIZATION}

\section{Disruption of Ligamentous Structures}

The ventral ligamentous structures such as the ALL and PLL and the anulus fibrosis provide significant stability to the spine. Any disruption of the ALL and PLLs, which may occur due to pathological processes or iatrogenic injury, can ultimately lead to loss of spinal integrity and subsequent instability. During preoperative planning, quantifying the degree of exposure-related disruption of ventral ligamentous structures is subjective and difficult to determine. Furthermore, there is a lack of biomechanical laboratory information in this regard. Spine surgeons should have knowledge of the individual biomechanical characteristics of the ALL and PLL to assist in the surgical decision-making process.

The ALL is a strong ligament attached to the VB edges at each segmental level of the spine. In addition to the strong physical characteristics of the ALL, its position ventral to the IAR provides a moment arm that resists extension (Fig. 1). It is usually not significantly disrupted even when ventral exposures are required because of the extensive width of the ligament. One key biomechanical feature of the ALL is its ability to provide a tension bandlike effect, which is an especially important contributor to postoperative spinal stability. In anterior lumbar interbody fusion, ventral anulotomy may significantly disrupt the ALL, requiring the PLL and dorsal elements to provide adequate tension band-like effect.

The PLL has far less biomechanical strength than its anterior counterpart in all areas of the spine. The position of the PLL dorsal to the IAR provides a short moment arm and, in combination with its weak intrinsic mechanical 

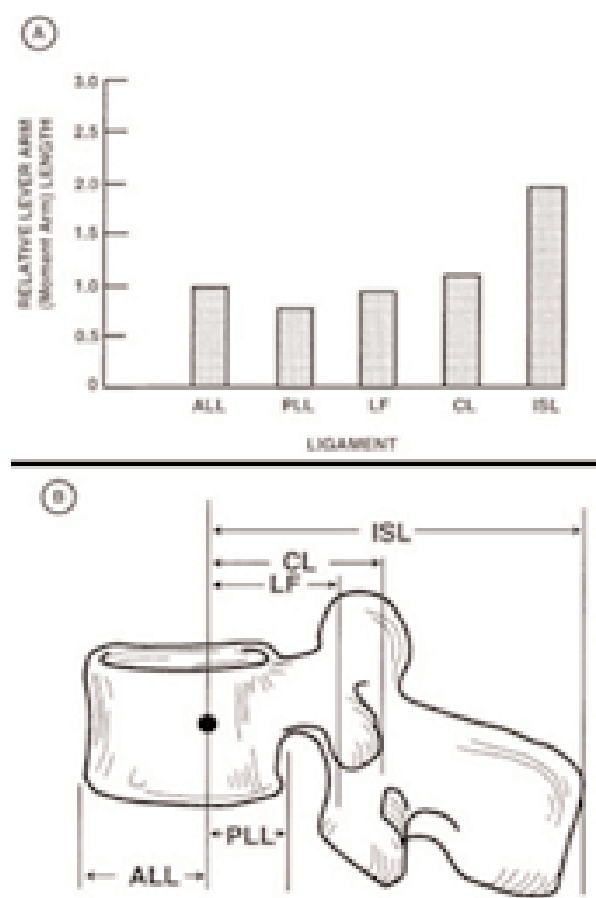

Fig. 1. A: Bar graph showing the relative lever arm (moment arm) length stratified by ligament causing flexion (or resisting extension). B: Drawing demonstrating the corresponding ligaments and their effective moment arms. The length depends on the location of the IAR. The dot represents the IAR. CL = capsular ligament; ISL = interspinous ligament; $\mathrm{LF}=$ ligamentum flavum.

properties, provides far less resistance to flexion than the dorsal elements (Fig. 1). The PLL is narrower in the midVB region and widens substantially in the region of the disc interspace. Therefore, a vertebrectomy in which the thecal sac is adequately decompressed at any level of the spine is certain to disrupt the PLL completely. The contribution of the PLL to resist flexion and distraction (albeit less than other ligaments) is impaired after many ventral surgical approaches in which the intention is to decompress the spinal cord.

Assessment of ligamentous stability during spinal decompression is essential. The application of spinal traction, interbody distraction, and other manipulative maneuvers can provide the surgeon with instant intraoperative feedback and a general sense of the integrity of ligamentous structures. The relative integrity of the spinal ligaments provides resistance to distraction, which is important when determining whether a strut graft can be used in a stand-alone manner. Excessive slackness of the ligamentous structures results in an inability of the abutting VBs to apply enough force to the strut graft to secure its position. Resistance to distraction provided by intact ligaments allows the VBs to "clamp down" on the strut graft (Fig. 2). Many spinal implants are placed in a distraction mode and rely on the intrinsic properties of the ligamentous structures to resist distraction (providing a tension band-like effect) to obtain optimal security of fixation. When assessing instability intraoperatively, however, the surgeon must keep in mind that the degree of laxity can easily be overestimated because of the effects of
A

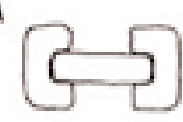

B

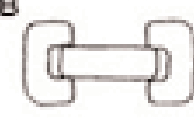

D

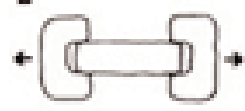

E
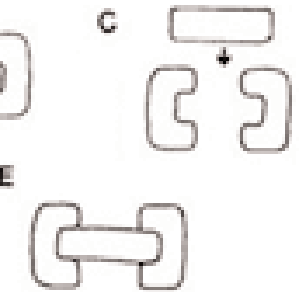

Fig. 2. A: A ventral interbody strut graft firmly positioned in relatively deep mortises. B: Ligamentous laxity results in an inability of the abutting VBs to apply enough force to the strut graft to secure its position. C-E: Distraction (C) followed by bone graft placement into well-formed mortises (D), followed by the relaxation of distraction (E). This provides the foundation for a well-conceived interbody fusion if adequate ligamentous resistance to distraction is present.

general anesthestic and paralytic agents, leading to the placement of unnecessary instrumentation.

Disc interspace disruption may also cause spinal instability ${ }^{19}$ (Benzel, et al., 1991, unpublished data; Chen, et al., 1999, unpublished data). The contribution of the anulus fibrosis to spinal stability, although significant, parallels that of the immediately adjacent ALL and the PLL. Its contribution cannot be separated from that of the ligaments. It should be stressed that the anulus fibrosis-ALLPLL complex contributes significantly to overall spinal integrity.

\section{BONE DISRUPTION}

Diminished integrity of the VB, whether due to the spinal pathological process ${ }^{13}$ or bone excision, reduces spinal stability. The amount of bone removed during ventral decompression clearly affects stability. Following complete vertebrectomy, the spine is clearly less intrinsically stable than one after partial VB resection. The portion of the VB, as well as the anatomical location of the part the VB resected, significantly affects spinal stability. A typical ventral cervical corpectomy will involve a partial VB "subtraction" to achieve appropriate spinal decompression over its entire rostrocaudal dimension (Fig. 3A). In addition, ventrolateral (Fig. 3B) and lateral extracavitary (Fig. 3C) decompressions also similarly involve incomplete removal of the VB over the complete rostrocaudal dimension of the VB. The degree of the ventral spinal integrity that remains is determined by the portion of the bone remaining in the ventral component of the VB.

The degree of iatrogenic spinal destabilization is determined by the location and extent of the region involved in the vertebrectomy. To demonstrate this point, consider the VB to be a cube composed of 27 equal-sized cubical segments (Fig. 4A and B). This theoretical model assumes posterior spinal stability. Disruption of the middle horizontal third, as viewed in the sagittal plane, results in destabilization and kyphosis with loss of the anterior and middle columns of Denis ${ }^{5}$ (Fig. 4C and D). One the other hand, removal of the middle coronal plane does not cause instability, with only partial disruption of anterior and middle columns. Resection of the ventral portion (the anterior nine cubes) may result in loss of stability; however, the loss of the middle and dorsal thirds in the coronal 
(A)

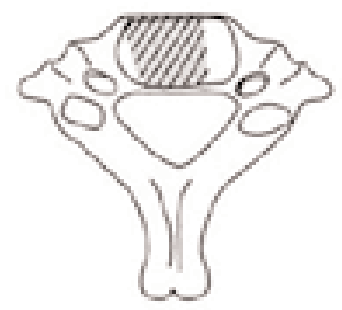

(B)

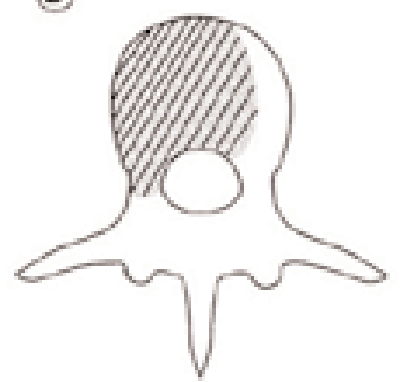

(c)

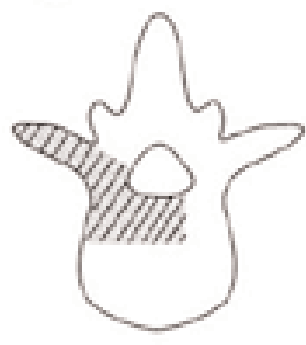

Fig. 3. Axial views of the extents of bone removal in a ventral cervical decompression (A), a ventrolateral thoracic or lumbar decompression (B), and a lateral extracavitary thoracic or lumbar decompression (C).

plane may not result in loss of spinal integrity if the ventral section of cubes is intact (Fig. 4E-H), the ALL remains intact, and dorsal column osseous and ligamentous integrity remains.

Minimizing the extent of ventral bone removal on one hand minimizes the effects of destabilization of the spine
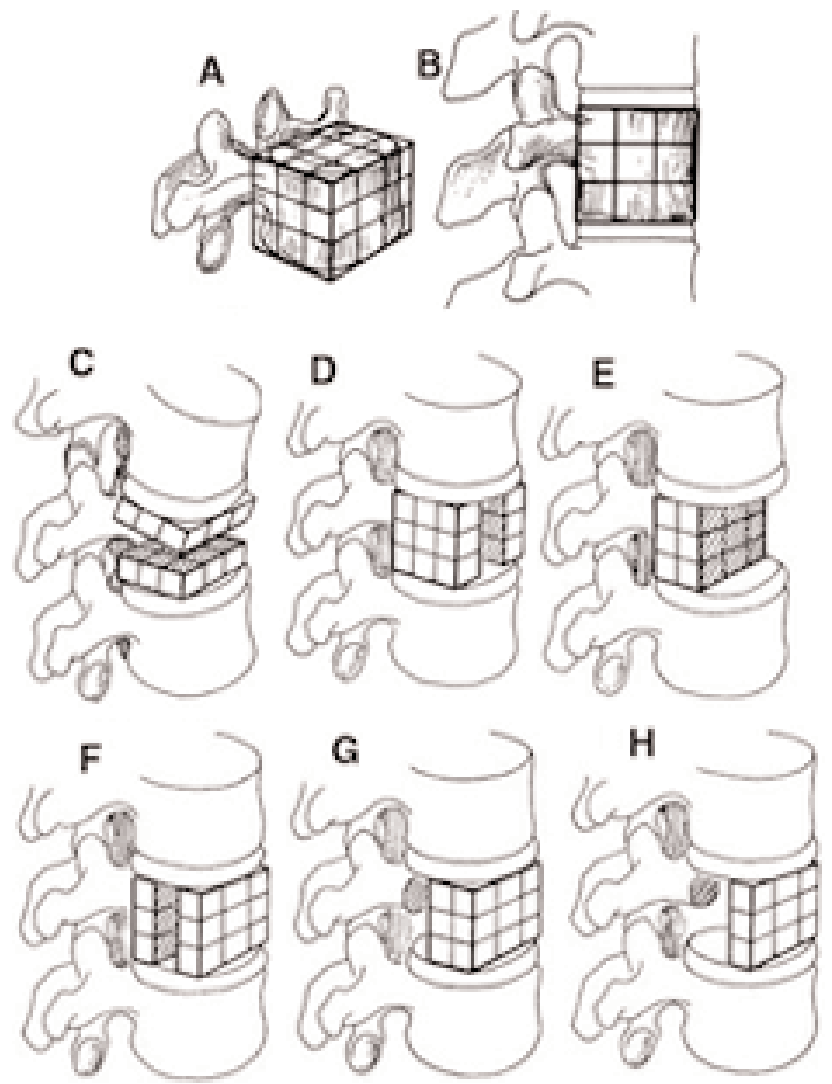

Fig. 4. A and B: The theoretical 27-cube model of the VB. C: Resection of the middle axial third of the VB. D: Resection of the midsagittal third of the VB; this resection does not significantly destabilize the spine. E-H: Partial vertebrectomy involving the removal of the ventral portion in the coronal plane of the VB (E) affects stability more than resection of the middle $(\mathrm{F})$ or dorsal portion $(\mathrm{G})$ of the VB in the coronal plane. H: Resection of the middle and the posterior thirds of the VB, in the presence of an intact posterior column, may not significantly disrupt spinal integrity. but on the other, may result in inadequate neural decompression (Fig. 5 left). The surgeon must attempt to overcome the natural tendency in ventral cervical surgery to waiver off the midline during decompression. A typical technical error involves the adequate decompression of the contralateral neural elements and insufficient decompression of the ipsilateral side (Fig. 5 right). An Erlenmeyer flask-like decompression (Fig. 6A) involves a narrow central decompression ventrally and widening of the decompression dorsally, thereby allowing for an adequate wide decompression without excessive osseous element excision. Furthermore, a relatively narrow ventral vertebrectomy allows for a more snug fit for the subsequent bone graft by providing greater lateral support (Fig. 6B).

During a lateral extracavitary approach, the cubic segmental model is useful (Fig. 7A). Thecal sac decompression should involve the most dorsal plane (the dorsal nine cubes) only on the side of exposure (Fig. 7B). Resection should not include the most ventral portion (ventral nine cubes) if iatrogenic destabilization is to be minimized. Using this hypothetical model, the ventral plane should be left intact and the middle plane used for interbody strut graft placement (Fig. 7C). The biomechanical advantage to placing the strut graft in the middle plane is that it is in line with the IAR and therefore in optimal position for axial load bearing by the strut graft. A concern regarding placement of a strut graft in the middle plane, however, is the limited room available for the graft's large surface area. Smaller grafts have less surface area and are more

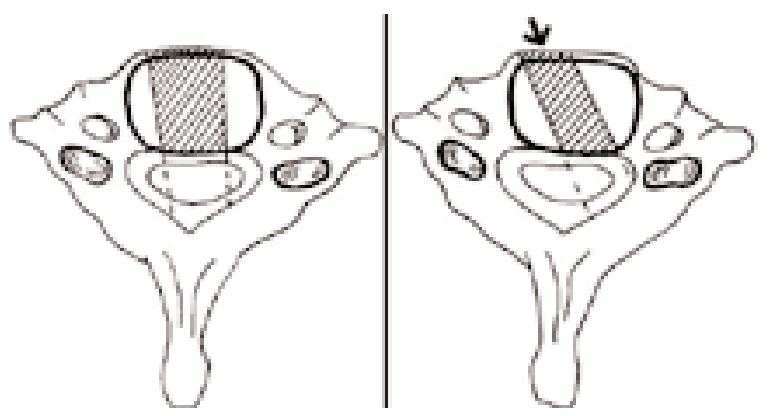

Fig. 5. Left: A narrow cervical corpectomy. Right: The end result of the surgeon's tendency to waiver from midline, erring toward decompression of the contralateral side and inadequate decompression of the ipsilateral side. 


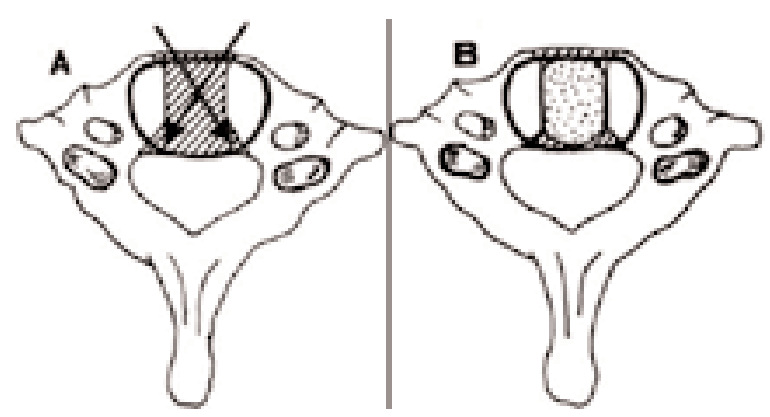

Fig. 6. A: Axial view of the Erlenmeyer flask-like exposure of the spinal canal, achieved by viewing the spine from both sides of the patient. B: Minimizing the width of the ventral portion of the trough enhances stability by minimizing bone removal and allowing for a snug fit for the bone graft, providing lateral stability for the strut graft by means of a buttressing effect.

prone to pistoning. One must weigh the potential benefits of minimizing destabilization with preservation of the anterior plane with the potential risks of graft subsidence that may occur with smaller struts.

Whereas the VBs provide ventral support and resistance to axial loads, other osseous structures, such as the uncovertebral joints, provide other vital biomechanical roles. The major biomechanical functions of uncovertebral joints include regulation of extension and lateral bending motion and torsion resistance. ${ }^{11}$ Destruction of the uncovertebral joints during surgical procedures or by neoplastic lesions (especially the posterior uncovertebral joints) can result in loss of those resistive forces.

\section{DORSAL SURGICAL SPINAL DESTABILIZATION}

Iatrogenic destabilization resulting from a dorsal ap-

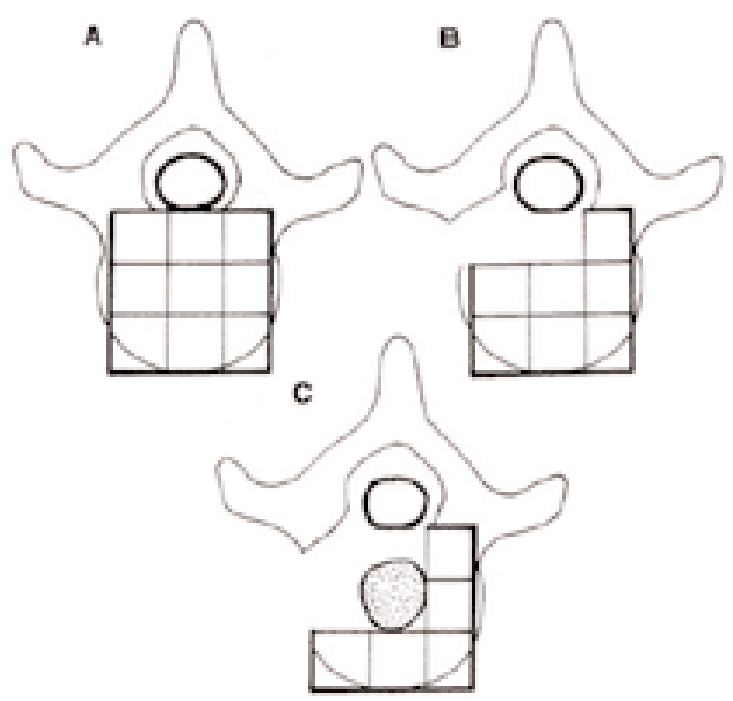

Fig. 7. A lateral extracavitary compression and the 27-cube model. A: Preoperative view. B: Resection of osseous components in the dorsal aspect of the VB preserves spinal integrity. $\mathrm{C}$ : Further ventral removal of bone is required for placement of a strut graft.
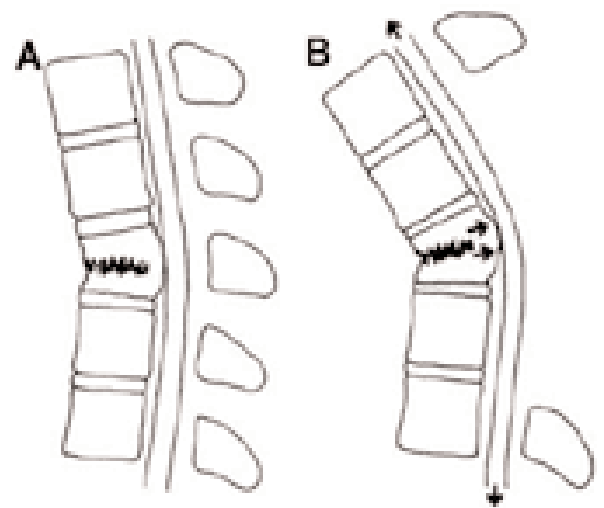

Fig. 8. Exaggeration of flexion deformity following laminectomy. A: Preoperative view. B: Postoperative sagittal view.

proach may be the end result of many factors. Inadequate appreciation of ventral spinal integrity may result in worsening of anterior and/or middle column VB injury following laminectomy due to disruption of the dorsal column. A slight increase in a flexion deformity created by the laminectomy-related destabilizing effects may occur after surgery in patients with worsening flexion deformity. When kyphosis worsens, a ventral mass lesion may act as a fulcrum, with resultant focal neural distortion (Fig. 8). The creation of a sharp angulation of the dural sac at the limits of a laminectomy may also occur during decompression. Laminoplasty may allow for the preservation
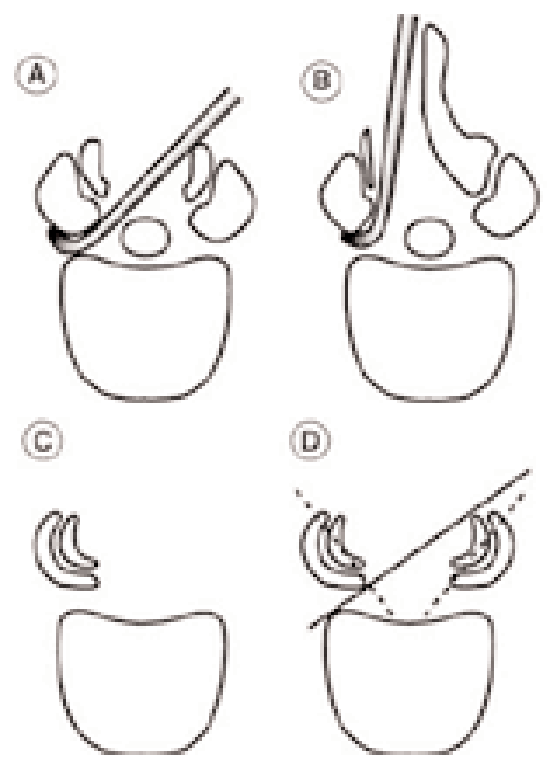

Fig. 9. A: Surgical trajectory provided by a laminectomy provides lateral recess access and decompression without significant facet joint and spinal destabilization. B: A Laminotomy provides a more vertical access, which is obstructed by the spinous process requiring greater facet resection (compared with laminectomy). C: The cup-shaped geometry of the facet joint must be considered. D: Preservation of the ventral portion of the cup should assist through the use of an appropriate surgical trajectory (solid line) in achieving postoperative stability by minimizing facet joint resection. Dashed lines depict facet joint angle. 


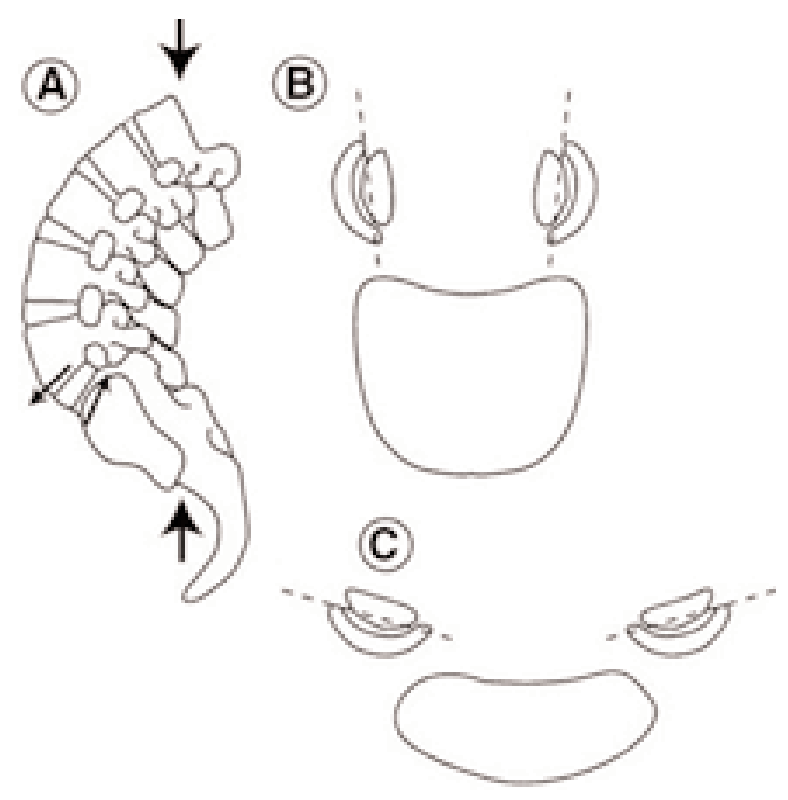

Fig. 10. A: The low lumbar and lumbosacral spine is subjected to significant translational loads if large axial loads are applied. B: Sagittally oriented facet joints (L4-5) resist translation poorly. C: Coronally oriented facet joints (L5-S1), however, provide good translational resistance.

dorsal tension band, thus potentially limiting one negative effect of laminectomy ${ }^{1}$ (Edwards and Heller, 2000, unpublished data).

Resection of the interspinous ligament may also affect spinal stability. Although the interspinous ligament is relatively weak compared with other ligaments, it has biomechanical advantages related to its long moment arm (Fig. 1). The surgeon must remember that this ligament is deficient at L4-5 and usually absent at L5-S1.

Excessive facet joint or pars interarticularis disruption during dorsal decompression may cause instability. In the cervical spine, approximately one third to one half of the facet joint may be resected without causing destabilization. ${ }^{15}$ Stability associated with lumbar facet disruption is typically "glacial" (slowly progressive spinal instability), which does not promote a significant risk for rapid development or progression of translational deformities. Lumbar facet integrity, however, may be minimally disrupted during laminectomy if an optimal trajectory is used., ${ }^{4,12,14,21}$ The trajectory provided by laminectomy allows for access to the lateral recess and decompression without necessitating facet joint destabilization (Fig. 9A). Laminotomy provides a vertical orientation for decompression and more limited lateral recess access because the spinous process is obstructed (Fig. 9B). The relative horizontal orientation of the facets, combined with their cuplike shape, creates an advantage for the surgeon during decompression if the facet anatomy is appreciated (Fig. 9C and D).

A combination of a vertically oriented facet joint and an exaggerated lordotic posture predisposes the lumbar spine to translational deformation. The relatively vertical orientation of the disc interspace in the lower lumbar region causes an allied axial load to result in the application of shearing force to the spine (Fig. 10). Sagittally oriented fa-

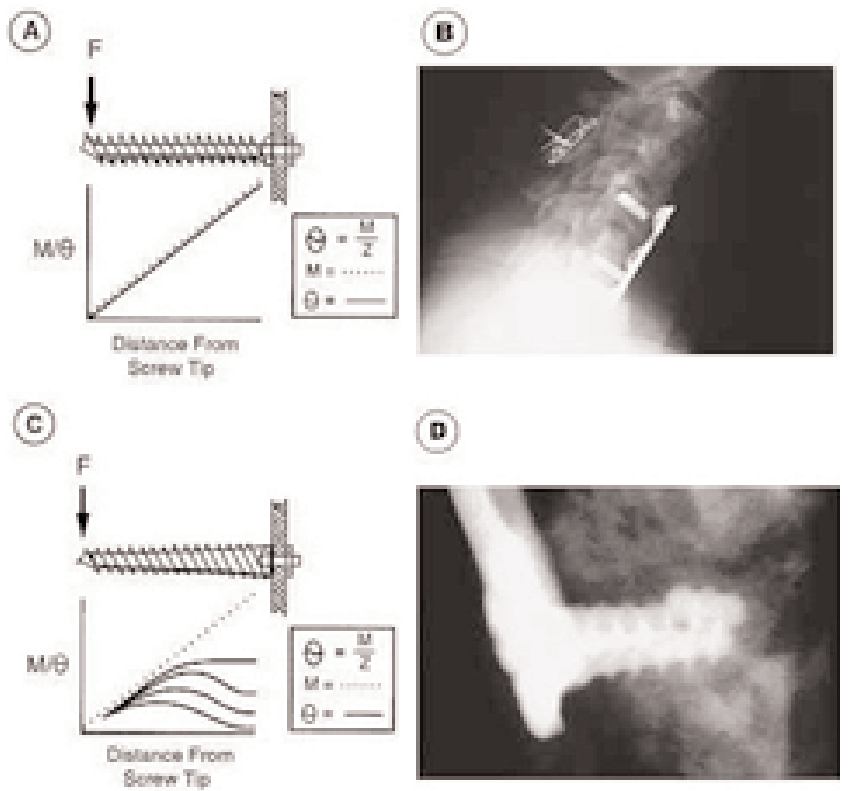

Fig. 11. A fixed-moment arm cantilever beam fixed innerdiameter screw is more likely to fracture at the screw-plate junction, and a conical inner-diameter screw is more likely to fracture midshaft if transverse loads are applied to the tip of the screw. This is so because the stress applied is maximum at the point of fracture, as depicted by line drawing in $\mathrm{A}$ and $\mathrm{C}$ and radiographically in $\mathrm{B}$ and D. The dotted lines represent the bending moment $(\mathrm{M})$. The solid line $(s)$ represent the strength. For tapered screws, a family of curves is depicted. The shape of the curve depends on the extent of taper of the screw's inner diameter $(\mathrm{C})$. ( $\mathrm{Z}=$ section modulus)

cet joints resist translation poorly, which is most evident at L4-5. The L5-S1 joint is relatively coronally oriented and resists translation well. Patients with injuries due to translational applied forces may benefit from fusion and instrumentation if laminectomy is performed and, particularly, if further facet joint disruption occurs at the time of surgery.

\section{BIOMECHANICS OF CONSTRUCT FAILURE}

\section{Failure of the Implant}

At points of maximum stress, $(\Theta)$ application spinal implants are most prone to fail. Stress $(\Theta)$ is a function of bending moment $(\mathrm{M})$ and the section modulus $(\mathrm{Z})$ and is defined as the equation $\theta=\mathrm{M} / \mathrm{Z}$. The section modulus defines the ability of an object such as a screw or a rod to resist bending. The section modulus $(\mathrm{Z})$ is an indicator of the strength of an object and is defined by the following equation $\mathrm{Z}=\mathrm{Pi} \times \mathrm{D}^{3} / 32$. The section on modulus is therefore proportional to the third power of the diameter of a rod or the third power of the inner diameter of a screw. Fixed-moment arm cantilevered screws may have a constant inner diameter or may have a tapered/conical inner screw diameter (Fig. 11). The point of maximum stress (the point of failure) of a fixed-inner diameter fixedmoment arm cantilever screw is usually at the screw-plate juncture. The point of failure of a tapered inner-diameter screw is between the tip of the screw and the plate. ${ }^{8}$ The section modulus remains unchanged along the length of 

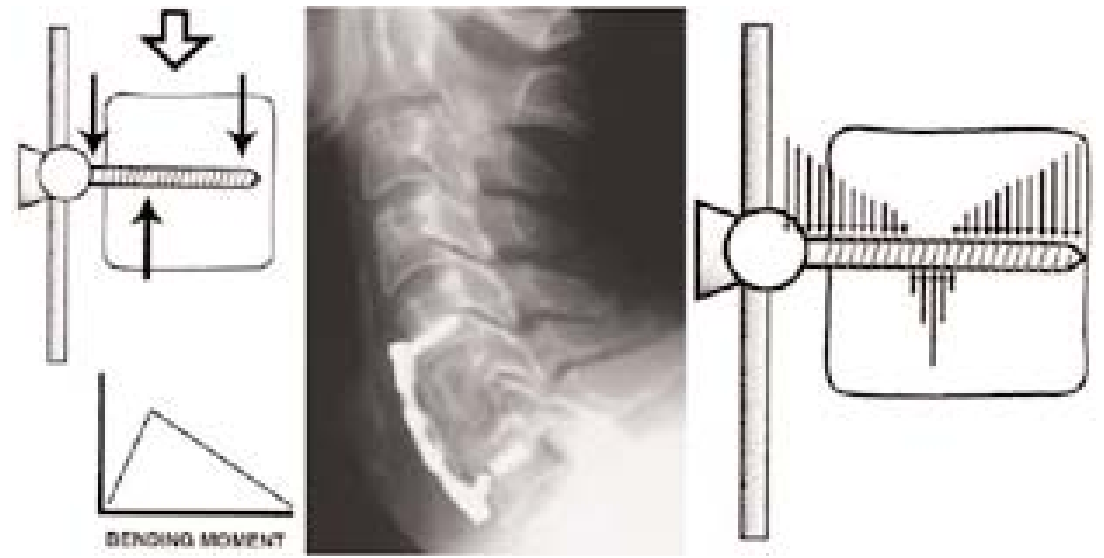

Fig. 12. Left: The three-point bending forces applied to a nonfixed-moment arm screw cantilever beam (closed arrows) exposed to axial loads (open arrow). These forces are applied in opposing directions. This is related to the differences in integrity of the materials through which the screw passes (for example, cortical bone, cancellous bone, metal) and the resistance to the loads applied. The bending moment (depicted below the line drawing) is maximum at the fulcrum. Center: Radiograph revealing a screw fracture resulting from such a mechanism. Right: The relative forces that a screw may "see" are situated in a transverse (perpendicular) orientation to the screw, as depicted. The force magnitude is proportional to the length of the arrows.

the fixed inner-diameter screw but rises exponentially for a tapered inner-diameter screw.

Nonfixed-moment arm cantilever screws are exposed to forces different from those associated with fixed-mo-

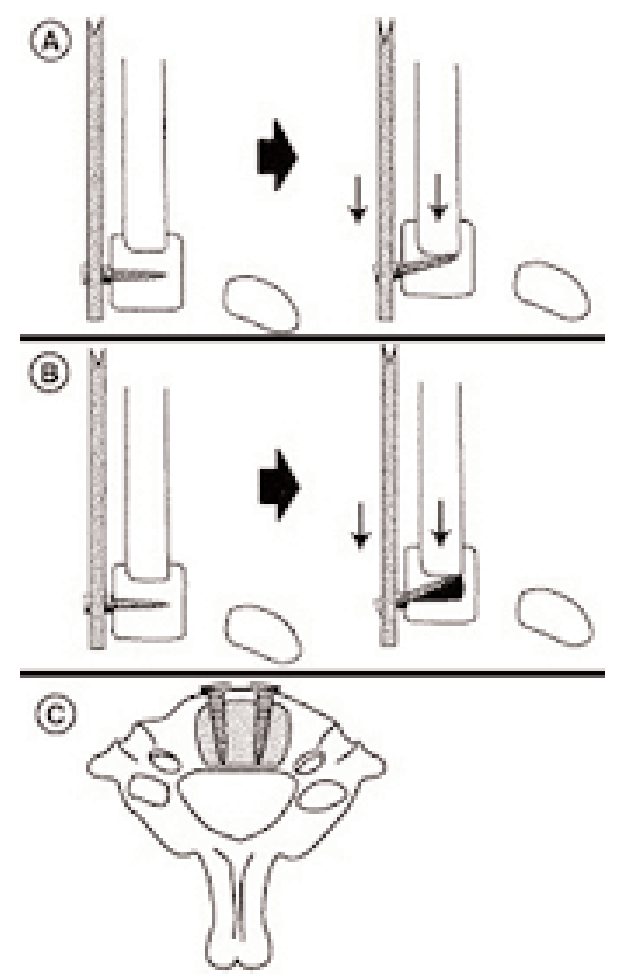

Fig. 13. A: Ventral nonfixed-moment arm cantilever beam screws that permit subsidence through toggling may abut the strut graft. B: This process results in degradation the VB bone (shaded area). C: Furthermore, the screws may partially obscure the strut-endplate interface, thus diminishing the possibility of solid arthrodesis. ment arm counterparts. Moment arms are perpendicular to fixed-moment arm screws (Fig. 11). On the other hand, nonfixed-moment arm screws are subjected to three-point bending moment forces (Fig. 12 left and center). As an axial load is applied to a nonfixed-moment arm cantilever beam screw/plate construct, the screw receives different force vectors, in magnitude and direction, at various points (Fig. 12 right). This is related to the differences in integrity of the materials through which the screw passes and resistance of the loads applied. The bending moment is maximum at the fulcrum, that at which point screw fracture is most likely to occur.

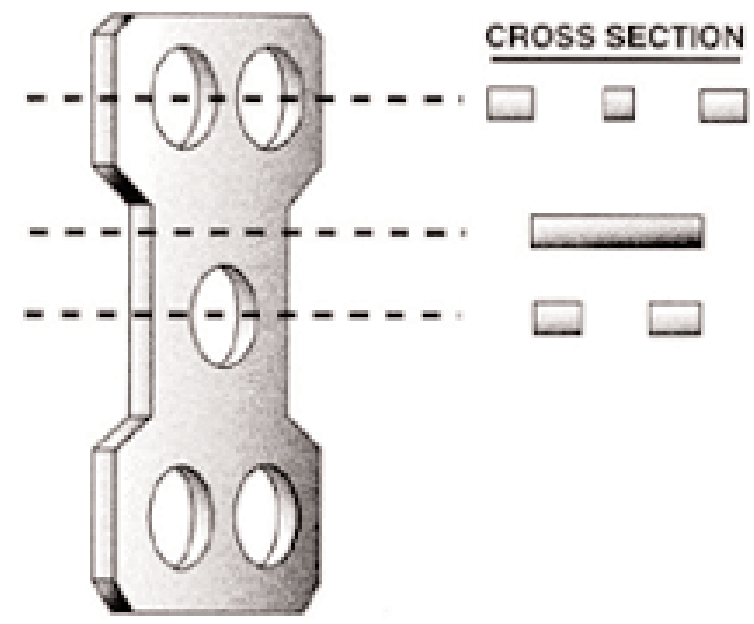

Fig. 14. A plate's strength depends on its geometry, as well as its cross-sectional area. In regions of smaller cross-sectional area, the section modulus is less (in general) than regions in which the cross-sectional area is greater. Although a plate appears to be very strong, it is no stronger than its weakest link. It is, indeed, most vulnerable at the site of greatest bending moment application. If it is weak (decreased strength or section modulus) at this point, fracture-related failure may occur. 

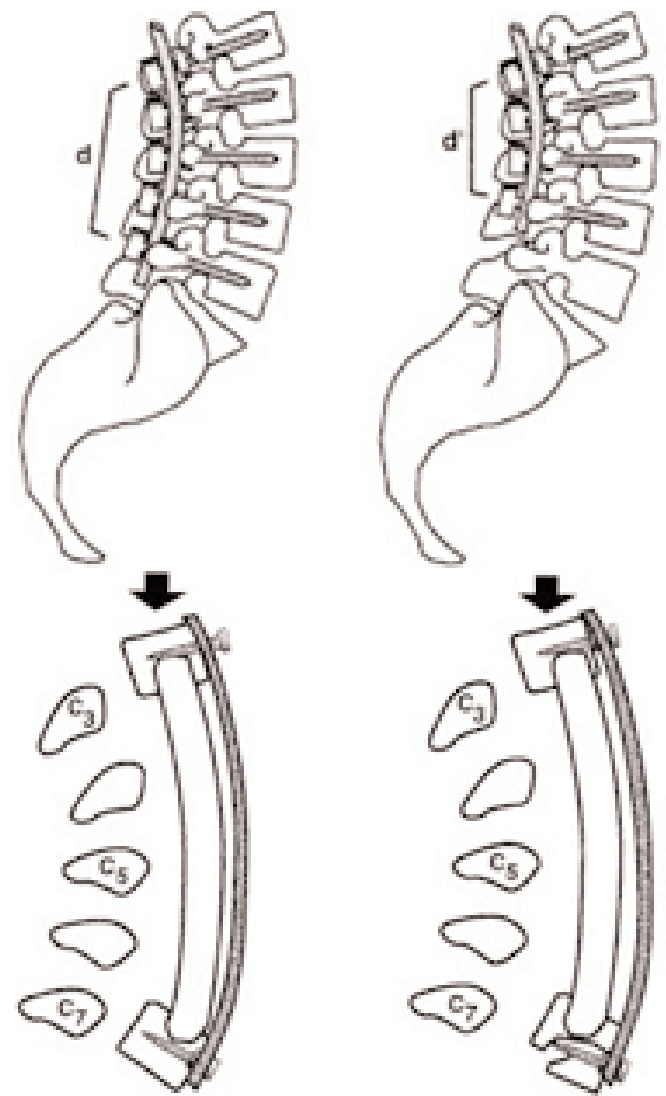

Fig. 15. Upper: Long rigid (fixed-moment arm cantilever beam) screw/rod multisegmental fixation tends to place greater load on the most caudal screws than the rostral screws, as depicted in a clinical example. In this situation, the use of a construct that extends one fewer segment caudally would have applied a lesser moment arm ( $\mathrm{d}$ and $\mathrm{d}^{1}$ ) and a lesser bending moment. Lower: The lower or most caudal portion of an implant is exposed to the greatest stress with respect to the cervical spine. Note the implant and strut kickout (kickthrough) occurred at the caudal aspect of the construct. This is related to geometrical and mechanical (physical) factors.

Toggling is a phenomenon in which nonfixed-moment arm cantilever beam screw forces degrade the integrity of the bone, as well as the screw-bone interface, by means of a "windshield wiping" motion (Fig. 13). The screw itself may sweep and abut the bone graft-endplate interface, thereby decreasing the surface area of contact and potentially diminishing the chance of solid fusion.

The section modulus (Z), which determines plate strength, is a function of the cross-sectional area and geometry. Although a plate may appear to be very strong, it is no stronger than its weakest link (Fig. 14). Similar to screws, plates break at the point at which maximum stress is applied, which is the point at which the ratio of the applied bending moment and the section modulus (M/Z) are maximum. ${ }^{8,9}$

Long rigid (fixed-moment arm) multisegmental fixation constructs tend to load the more caudal screws far more than the rostral screws. Long fixed-moment arm cantilever beam screw implants are associated with a high failure rate (Bolesta and Rechtine, 1998, unpublished

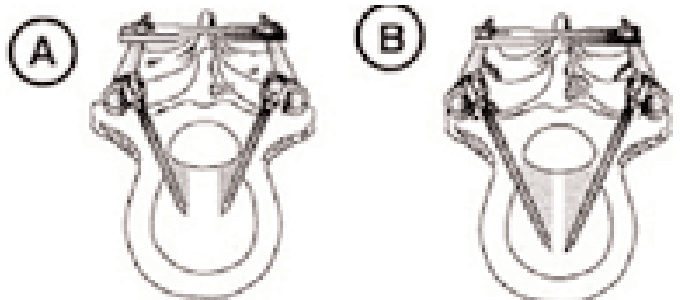

Fig. 16. The triangulation of pedicle screws provides additional resistance to pullout. A: Pullout resistance is proportional not only to volume of bone between screw threads, but also to the triangular area defined by the screw and the perpendicular and the dorsal VB surface (shaded area). B: Whereas screw length does not routinely contribute significantly to pullout resistance, it contributes significantly when screws are rigidly triangulated. Note the increase in shaded area. Increasing the screw angle (that is, toe-in) also increases the size of the shaded area and thus pullout resistance.

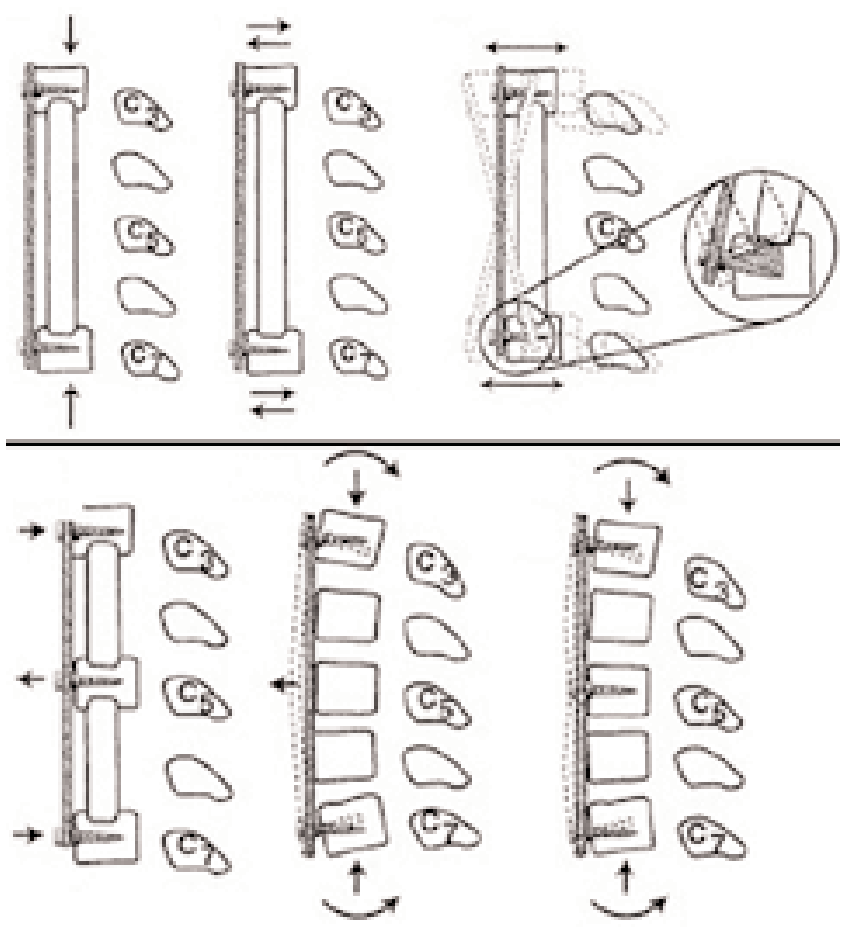

Fig. 17. A rigid fixed-moment arm cantilever beam implant involving two screws attached at each end. This is a bridging implant. Upper Left: This construct resists axial loads (vertical arrows) well. Loads, however, are usually applied from a variety of orientations. Upper Center: Shear loads (horizontal arrow) can cause translation. Upper Right: This may cause the screw-bone interface to degrade (shaded areas) and to ultimately fail, usually as a result of axial loading. Lower Left: The addition of a third (intermediate) point of fixation causes the implant to resist these loads more effectively (for example, translation) through a threepoint bending mechanism (arrows). The three-point bending effect resists shear-producing loads in multiple planes. Axial loads can also be resisted more effectively by using an intermediate fixation point. Lower Center and Right: The flexibility of an implant bearing an axial load (lower center) is significantly diminished using an additional intermediate point of fixation (lower right). The intermediate fixation point minimizes flexibility (dashed implant in lower center and right). 
(A)

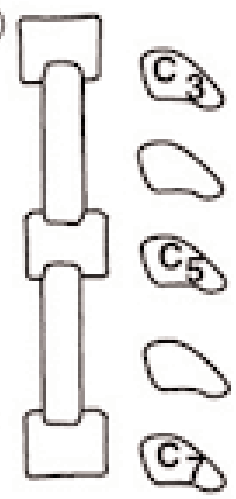

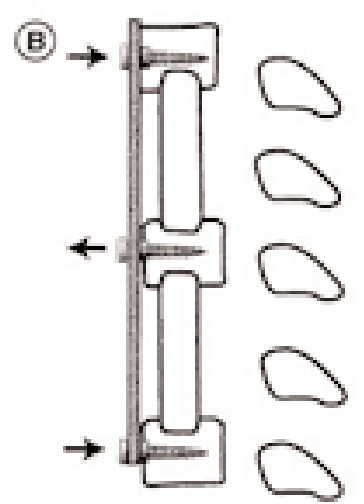

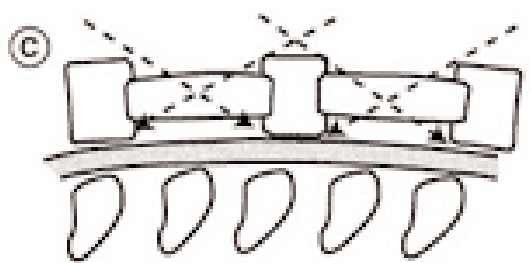

Fig. 18. A: A long C4-6 decompression can be accomplished via $\mathrm{C}-4$ and $\mathrm{C}-6$ corpectomies and a $\mathrm{C} 3-5$ and a $\mathrm{C} 5-7$ interbody fusion. $\mathrm{B}$ and $\mathrm{C}$ : This provides a solid VB site (C-5) for intermediate screw fixation (B), while providing adequate visualization for dural sac decompression (C, dashed arrows).

data). When clinically appropriate, the use of a shorterlength implant, with its shorter moment arm, may decrease the risk of caudal construct failure (Fig. 15 upper).

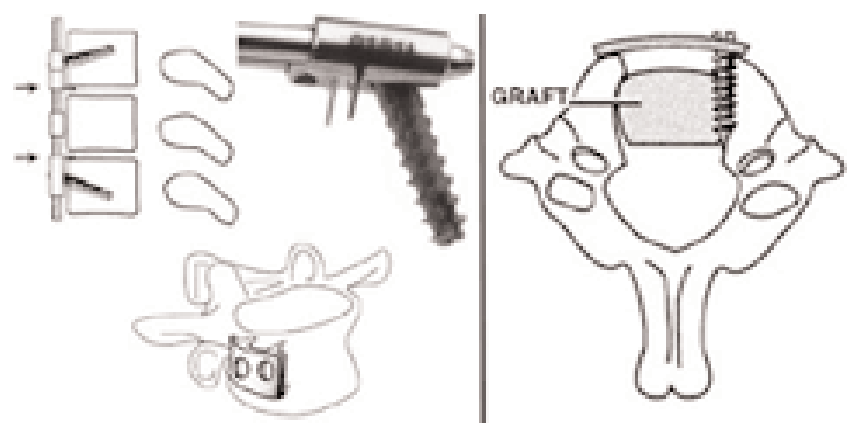

Fig. 19. Left: A buttress, in which a fin is applied at the endplate in the ventral VB cortex or a spike is placed into a VB provides "extra" points of fixation and hence security. A small (short) fin or spike is all that is required. A fin or spike need only pass through or past the cortical bone to confer advantage. This is portrayed using the fin of a DOC system that moves just past the cortical edges of the VB in the region of the endplate, thus taking advantage of the "boundary effect." The device is now capable of more effectively bearing axial loads (arrow). Right: An interference screw may be inserted between the bone graft and a corpectomy wall (parent $\mathrm{VB}$ ) in procedures involving a long ventral cervical corpectomy fusion and instrumentation. Note the purchase by the screw at both the parent VB bone and the bone graft. In addition, the screw may force the bone graft toward the contralateral side of the corpectomy trough, thus providing increased integrity and VB-bone graft contact. Care must be taken to not retropulse the bone graft. This strategy provides augmented fixation to the intermediate VBs.
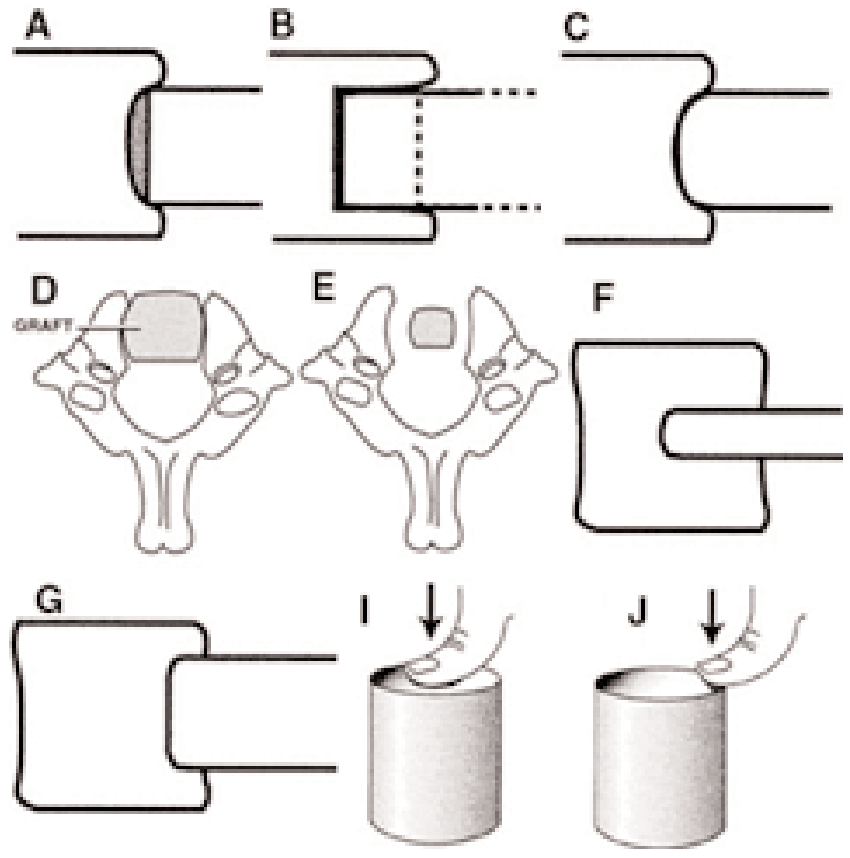

Fig. 20. A: A square (flat)-ended bone graft does not fit well into a rounded VB mortise. Nonunion may occur as a result of an inadequate surface area of contact (gray area). B: Alternatively, subsidence, which can not be desirable, may occur because of the inability of the VB mortise to prevent "penetration" by the ill-fitted strut graft. C: Maximizing the surface area of contact and the closeness of fit between the bone graft and the VB minimizes concentration of stress and the chance of nonunion or excessive subsidence. D and E: Interbody bone-bone interface failure (cervical spine) can be minimized by optimizing the lateral fit of the bone graft with the corpectomy trough wall. D: This creates a lateral buttressing effect. Lateral bone healing at intermediate VB segments is encouraged by this process (D). If the bone graft is smaller in diameter, it is suspended in space, thus eliminating the aforementioned buttressing and healing advantage $(\mathrm{E})$. F and $\mathrm{G}$ : The surface contact area between the bone graft and the accepting bone (VB) is inversely proportional to the extent of penetration (subsidence) of the bone graft. A smaller surface area of contact (F) resists penetration less well than larger surface area of contact $(\mathrm{G})$. $\mathrm{H}$ and I: If a thumb is used to apply force to the center of the end of a tin can $(\mathrm{H})$, the extent of depression is greater than if a similar force is applied to the edge of the can (I). This is analogous to a force applied to the center of an endplate compared with the VB-endplate region along the VB cortex wall. This is due to a relative increased integrity of the $\mathrm{VB}$, in terms of its ability to bear axial loads at its edge. This phenomenon is known as the "boundary effect." The cortical margin along the ventral surface of the VB is a very effective buttress that can be used to employ the "boundary effect" to a clinical advantage.

Long constructs that end in the lower cervical region, as a result of the geometrical configuration of this area, place much stress in the caudal screw-bone interface (Fig. 15 lower) (Sasso, et al., 2000, unpublished data).

\section{Failure of the Implant-Bone Interface}

Optimization of the integrity of the implant-bone interface can reduce the incidence of instrumentation failure. The surgeon should attempt to distribute loads such that no single portion of the implant or spine bears an exces- 


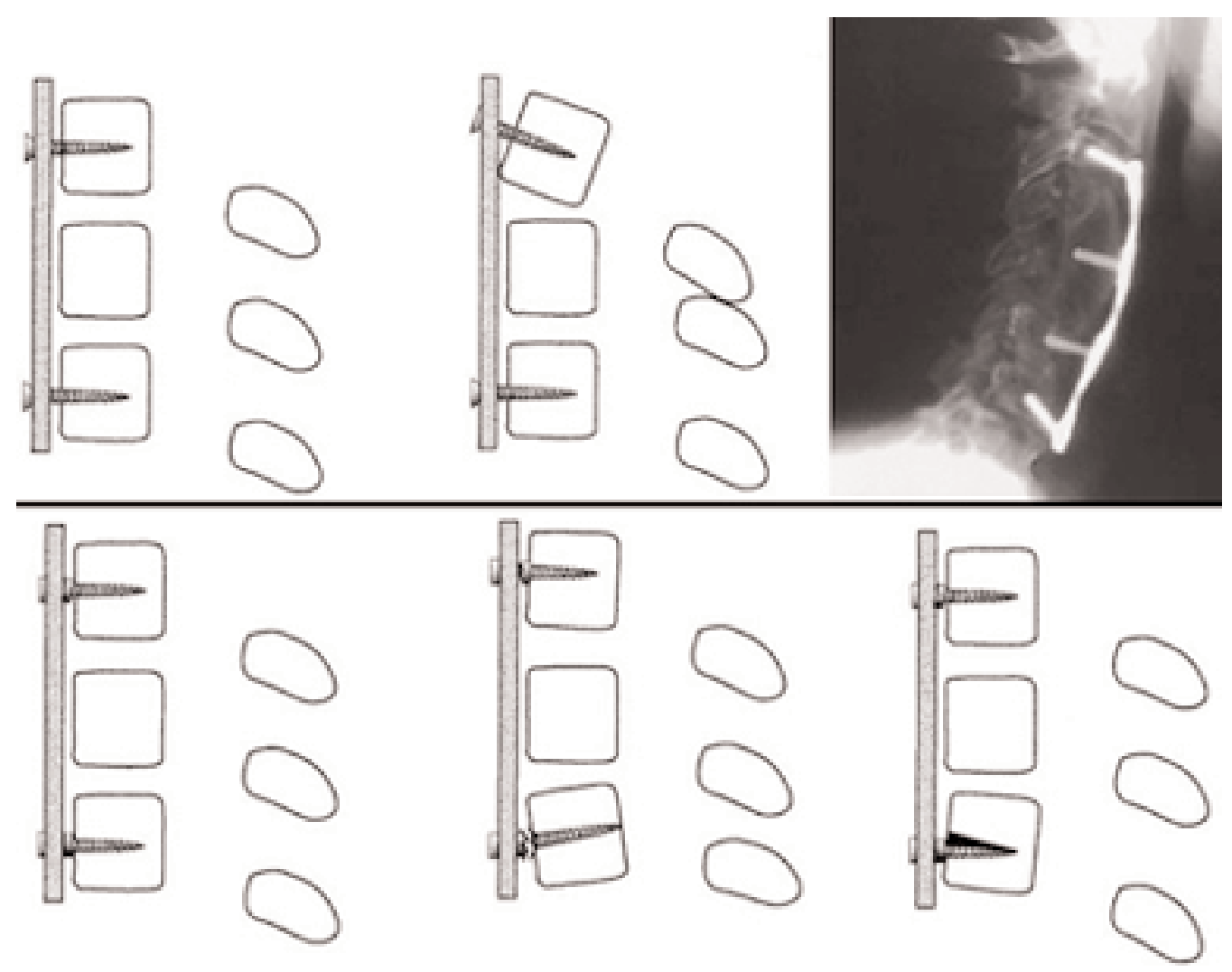

Fig. 21. Upper Left and Center: The toggling that is permitted by nonfixed-moment arm cantilever beam implants permits angular deformation. This is not usually considered desirable. Upper Right: Radiograph demonstrating a clinical example of angular deformation. Lower: Fixed-moment arm cantilevers (lower left) may fail, either by fracture (lower center) or by failure at the screw-bone interface (lower right, shaded area), resulting in angular deformation, as depicted.

sive portion of the load. This can be accomplished by improving the integrity of the existing implant-bone interfaces, providing additional implant-bone interfaces, improving the integrity of the bone, and by normalizing the geometry. Offloading the implant, by use of dynamic implants, allows more load sharing through the bone graft ${ }^{2}$ and has been shown to minimize the risk of hardware failure. $^{7}$

\section{Improving the Existing Implant-Bone Interface}

Minimizing screw pullout involves requires an understanding of screw-related anatomy and geometrical placement. The important anatomical aspects of a screw include the head, core, thread, and tip. Screw pullout is mainly a function of the volume of bone between the threads. Alteration of thread pitch and distance between threads affect the interthread bone volume. Triangulation of pedicle screws provides additional resistance to pullout. Pullout resistance is proportional not only to the volume of bone between screw threads but also to the triangular area defined by the screw: the perpendicular and dorsal VB surface (Fig. 16A). Whereas screw length does not contribute significantly to pullout resistance, rigidly triangulated screws significantly affect resistance. Increasing the screw angle (toe-in) also increases the aforedescribed triangular area and thus pullout resistance (Fig. 16B).
Additional strategies include expanding the screw tip and undertaking screw hole augmentation strategies, including the use of bone chips and polymethylmethacrylate. Appreciating the dynamic angular relationships of the lower cervical spine at the cervicothoracic junction is crucial. Considerable loading of the caudal screw-bone junction at the lower cervical spine often leads to failure (Sasso, et al., 2000, unpublished data). This high failure rate at the ventral cervicothoracic region is in part related to the angle of the screw in relation to the axis that is perpendicular to the floor (Fig. 15 lower).

\section{Providing Additional Implant-Bone Interfaces}

The integrity of a spinal construct is proportional to the number of high-quality fixation points. Additional fixation points provide a significant biomechanical advantage. A rigid fixed-moment arm cantilever beam implant involving two screws attached at each end acts as a bridging implant that must resist spinal deformation in multiple planes (Fig. 17 upper left and center). This construct will resist axial loads well while the strut graft shares the load with the implant. This construct, however, may not adequately resist translational forces, resulting in degradation of the screw-bone interface and subsequent failure due to axial loading (Fig. 17 upper right). The longer the length of the implant, the more prone it is to these effects. The 


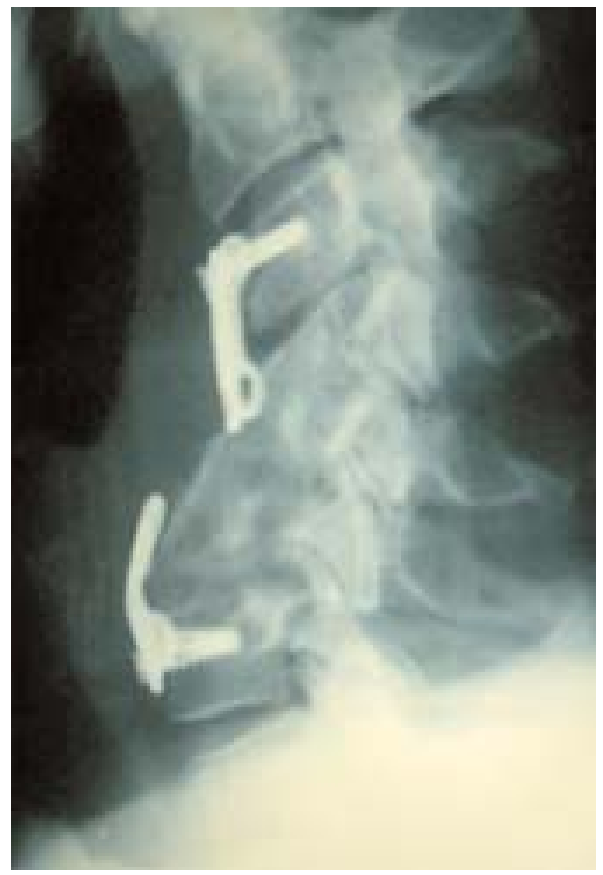

Fig. 22. An advantage of kickplates is that they permit axial deformation; however, they do allow unwanted angular deformation and graft dislodgment.

addition of a third intermediate point of fixation results in greater resistance to translational loads via a three-point bending mechanism (Fig. 17 lower left). The intermediate point of fixation will also increase the resistance to axial loads. The extent of deformation of a flexible implant is limited by the use of an intermediate point of fixation (Fig. 17 lower center and right). Intermediate points of fixation are most useful if placed into the VBs. Screws placed into the bone graft decrease the overall stability of the construct and weaken the graft itself. Minimization of platerelated bowing is not impeded by an intermediate screw placed into the bone graft. ${ }^{6}$ To the contrary, it causes potentially detrimental ventral and dorsal loads to be placed on the graft itself.

In multilevel cervical corpectomy, an intermediate point of fixation can be obtained by leaving the intermediate VB in place. For example, one could leave the C-5 VB intact when undertaking a C4-6 decompression and C3-7 fusion, thus using two interbody fusions (C3-5 and C5-7) instead of a long C3-7 fusion (Fig. 18). By harnessing the biomechanical advantage conferred by threepoint fixation, one avoids increasing the number of interfaces that must undergo arthrodesis. Another possible means of achieving the same biomechanical advantage is placing a single strut graft ventrally and supplementing it with lateral mass fixation dorsally. This, however, requires a second operation. It has been shown that three- or fourlevel cervical corpectomies may be effectively augmented with posterior fixation. ${ }^{18}$

Implant-bone interface integrity may also be optimized by the addition of spikes and the use of a buttress point of fixation at the endplate in the region of the cortical margin; this adds other fixation points of biomechanical advantage (Fig. 19 left). An interference screw between the bone graft and a corpectomy may be placed in a longlength ventral corpectomy fusion and instrumentation procedure. An interference screw captures two separate and juxtaposed osseous surfaces (Fig. 19 right). This technique provides a contact surface between the parent bone and screw while "gripping" the bone graft. In addition, the screw may force the bone graft toward the contralateral side of the corpectomy trough, thus increasing the integrity and VB-bone graft contact. Care must be taken not to retropulse the bone graft. When using interference screws in conjunction with an anterior cervical construct, it is important to lock the screw head to the cervical plate to prevent backout if bone resorption occurs.

\section{Improving Bone-Bone Interface Failure}

The interface between the bone graft and VB is certainly prone to failure. Subsidence-related surgical complications can be minimized by conducting proper "carpentry" techniques that involve bone shaping and fitting. The creation or shaping of a mortise in the VB and the precise fitting and shaping of an interbody bone graft are vital to minimize the chance of dislodgment or subsidence. Factors affecting the incidence and extent of subsidence include the closeness of fit of the bone graft in the VB mortise, the surface area of contact between the bone graft and VB; and the character or quality of the contact surfaces. A square flattened bone graft does not fit well into a rounded VB mortise (Fig. 20A-C). A poor fit increases the risk of nonunion because of inadequate surface area contact or excessive subsidence caused by the concentration of stresses and loads at the VB-bone graft interfaces. Lateral fit of the bone graft against the wall of the corpectomy trough further optimizes interbody bone-bone interface relationships. Creating a lateral buttressing effect can help minimize bone-bone interface failure (Fig. 20D and E). The surface contact area between the bone graft and the VB is inversely proportional to the extent of subsidence. Increasing bone-bone contact surface area will decrease the risk of graft pistoning (Fig. 20F and G). The extent of endplate preservation and proximity of the contact point to the edge of the VB affects the quality of contact surfaces. The ventral VB provides a significant advantage regarding a buttressing effect because it bears axial loads better than the softer cancellous portion of bone. This is akin to comparing it to the edge of a tin can with regard to load-bearing ability (Fig. $20 \mathrm{H}$ and I). This is substantiated by biomechanical data indicating greater construct strength when the cortical portion of a graft is positioned in line with the cortical surface. ${ }^{20}$ Some implants take advantage of the boundary effect by providing support at the edge of the VB, thus maximizing axial-load resistance. The greatest biomechanical advantage regarding interbody axial load-bearing ability is achieved when a strut graft is nearly the same size as the VB with respect to the contact surface areas.

The concept of spinal implant dynamism permits deformation in a controlled manner, while not permitting undesirable deformation. The advantage of using deformable implants is that they allow angular and/or axial deformation. Angular deformations may be permitted by the use of nonfixed-moment arm cantilever constructs (Fig. 21 upper) by tension band devices, ${ }^{10}$ and by failure of fixed 
moment are cantilevers (Fig. 21 upper). Axial deformation is permitted by slotted plates, collapsible plates, screws that slide on plates or rods, or sliding platforms. This takes advantage of early subsidence that occurs predominantly in the first 2 weeks following placement of a strut graft. ${ }^{3}$ Kick plates have the advantage of permitting axial deformation, allow angular deformation, and have been associated with catastrophic outcomes after graft dislodgment (Fig. 22). ${ }^{17}$

\section{Improving Bone and Bone-Implant Integrity}

Bone-implant integrity may be enhanced at the time of surgery to improve the integrity of screw purchase. Tapping cortical bone increases the integrity of the screwbone interface, whereas tapping cancellous bone weakens it. Drilling cancellous bone without tapping provides greater pullout resistance than tapping. The use of an awl during hole preparation improves pullout strength by pushing and compressing the cancellous bone laterally. Other techniques such as placing bone chips or the use of pressurized polymethylmethacrylate can augment screwbone integrity especially in osteoporotic bone.

\section{CONCLUSIONS}

A surgeon's appreciation of the biomechanical properties of the ligamentous and osseous structures of the spine is vital to avoid destabilization during ventral and dorsal spinal exposures. Construct failure occurs as a result of failure of the implant, failure at the implant-bone interface, or failure of the component-component juncture. The vast majority of spinal implant failures, however, are not actually device failures but instead are surgeon-related errors in proper construct selection and appropriate patient selection. Having a clear understanding of biomechanical principles of spinal implants and how as well as why they fail will minimize the complications requiring reoperation.

\section{Acknowledgment}

All figures were previously published in Biomechanics of Spine Stabilization. ISBN 1-879284-82-0. AANS Publications. Benzel EC, author. 2001, pp. 526.

\section{References}

1. Baisden J, Voo LM, Cusick JF, et al: Evaluation of cervical laminectomy and laminoplasty. A longitudinal study in the goat model. Spine 24:1283-1289, 1999

2. Brodke DS, Gollogly S, Alexander MR, et al: Dynamic cervical plates: biomechanical evaluation of load sharing and stiffness. Spine 26:1324-1329, 2001

3. Carl AL, Tranmer BI, Sachs BL: Anterolateral dynamized instrumentation and fusion for unstable thoracolumbar and lumbar burst fractures. Spine 22:686-690, 1997

4. Darden BV Jr, Wade JF, Alexander R, et al: Far lateral disc herniations treated by microscopic fragment excision. Techniques and results. Spine 20:1500-1505, 1995

5. Denis F: The three column spine and its significance in the classification of acute thoracolumbar spinal injuries. Spine 8: 817-831, 1983
6. Dick JC, Jones MP, Zdeblick TA, et al: A biomechanical comparison evaluating the use of intermediate screws and crosslinkage in lumbar pedicle fixation. J Spinal Disord 7:402-407, 1994

7. Epstein NE: Fixed vs dynamic plate complications following multilevel anterior cervical corpectomy and fusion with posterior stabilization. Spinal Cord 41:379-384, 2003

8. Hollowell JP, Reinartz J, Pintar FA, et al: Failure of synthes anterior cervical fixation device by fracture of Morscher screws: a biomechanical study. J Spinal Disord 7:120-125, 1994

9. Kanayama M, Cunningham BW, Weis JC, et al: The effects of rigid spinal instrumentation and solid bony fusion on spinal kinematics. A posterolateral spinal arthrodesis model. Spine 23:767-773, 1998

10. Konno S, Kikuchi S: Prospective study of surgical treatment of degenerative spondylolisthesis: comparison between decompression alone and decompression with graf system stabilization. Spine 25:1533-1537, 2000

11. Kotani Y, McNulty PS, Abumi K, et al: The role of anteromedial foraminotomy and the uncovertebral joints in the stability of the cervical spine. A biomechanical study. Spine 23: 1559-1565, 1998

12. Muller A, Reulen HJ: A paramedian tangential approach to lumbosacral extraforaminal disc herniations. Neurosurgery 43:854-862, 1998

13. Oda K, Shibayama Y, Abe M, et al: Morphogenesis of vertebral deformities in involutional osteoporosis. Age-related, threedimensional trabecular structure. Spine 23:1050-1056, 1998

14. Osman SG, Nibu K, Panjabi MM, et al: Transforaminal and posterior decompressions of the lumbar spine. A comparative study of stability and intervertebral foramen area. Spine 22: 1690-1695, 1997

15. Raynor RB, Pugh J, Shapiro I: Cervical facetectomy and its effect on spine strength. J Neurosurg 63:278-282, 1985

16. Rechtine GR Jr, Cahill D, Chrin AM: Treatment of thoracolumbar trauma: comparison of complications of operative versus nonoperative treatment. J Spinal Disord 12:406-409, 1999

17. Riew KD, Sethi NS, Devney J, et al: Complications of buttress plate stabilization of cervical corpectomy. Spine 24: 2404-2410, 1999

18. Schultz KD Jr, McLaughlin MR, Haid RW Jr, et al: Singlestage anterior-posterior decompression and stabilization for complex cervical spine disorders. J Neurosurg (Spine 2) 93: 214-221, 2000

19. Wall EJ, Bylski-Austrow DI, Shelton FS, et al: Endoscopic discectomy increases thoracic spine flexibility as effectively as open discectomy. A mechanical study in a porcine model. Spine 23:9-16, 1998

20. Wang JC, Zou D, Yuan H, et al: A biomechanical evaluation of graft loading characteristics for anterior cervical discectomy and fusion. A comparison of traditional and reverse grafting techniques. Spine 23:2450-2454, 1998

21. Weiner BK, McCulloch JA: Microdecompression without fusion for radiculopathy associated with lytic spondylolisthesis. J Neurosurg 85:582-585, 1996

22. Zeidman SM, Ducker TB, Raycroft J: Trends and complications in cervical spine surgery: 1989-1993. J Spinal Disord 10: 523-526, 1997

Manuscript received July 28, 2003.

Accepted in final form August 19, 2003

Address reprint requests to: Richard P. Schlenk, M.D., Department of Neurosurgery, The Cleveland Clinic Foundation, 9500 Euclid Avenue, S80, Cleveland, Ohio 44195. email: benzele@ccf.org. 